\title{
ERROR ANALYSIS OF A BOUNDARY ELEMENT COLLOCATION METHOD FOR A SCREEN PROBLEM IN $\mathbb{R}^{3}$
}

\author{
M. COSTABEL, F. PENZEL, AND R. SCHNEIDER
}

Dedicated to Professor Dr. E. Meister on the occasion of his sixtieth birthday

\begin{abstract}
We examine the numerical approximation of the first-kind integral equation on a plane rectangle defined by the single-layer potential of the threedimensional Laplacian. The solution is approximated by nodal collocation with piecewise bilinear trial functions on a rectangular grid. We prove stability and convergence of this method in the Sobolev space $\widetilde{H}^{-1 / 2}$. A key ingredient in the proof is the observation that the collocation equations define symmetric positive definite Toeplitz matrices.
\end{abstract}

\section{INTRODUCTION}

Let $\Omega:=(-1,1) \times(-1,1) \subset \mathbb{R}^{2}$. The first-kind integral equation on $\Omega$,

$$
V u(x):=\frac{1}{4 \pi} \int_{\Omega} u(y) \frac{d y}{|x-y|}=f(x) \quad(x \in \Omega),
$$

gives the solution of the Dirichlet problem for the Laplace equation in $\mathbb{R}^{3} \backslash \bar{\Omega}$ with Dirichlet data $f$ given on $\Omega$ ("screen problem") (see [12, 25]). One important application of (1.1) has been the computation of the capacitance of the square plate $\Omega$. The numerical computations were frequently made with collocation methods, although a convergence proof was not available. For the numerical approximation of the solution $u$ of (1.1), we introduce a grid $\square^{N}$ on $\Omega$,

$$
\square^{N}:=\left\{x_{k}^{N}:=\frac{k}{N}=\left(\frac{k_{1}}{N}, \frac{k_{2}}{N}\right) \mid k \in \mathbb{Z}^{2}\right\} .
$$

As trial space we use the space $S^{1}\left(\square^{N}\right)$ of all continuous piecewise bilinear functions with nodes in $\square^{N}$. Then our collocation scheme is the following: Find a function $u^{N} \in S^{1}\left(\square^{N}\right)$ whose nodal values vanish outside $\bar{\Omega}$ such that

$$
V u^{N}\left(x_{k}^{N}\right)=f\left(x_{k}^{N}\right) \text { for } x_{k}^{N} \in \square^{N} \cap \bar{\Omega} .
$$

The main result of this paper is the stability and convergence of this method in the Sobolev space $\widetilde{H}^{-1 / 2}$ (see Theorem 3.1).

Received March 23, 1990.

1991 Mathematics Subject Classification. Primary 65N35, 65R20; Secondary 45L10.

The first and third authors were supported by the DFG-Forschergruppe Ko 634/32-1. 
The mapping properties of the operator $V$ in Sobolev spaces are well known (see [25]). In particular, $V$ defines a positive definite symmetric bounded bilinear form on the space $\widetilde{H}^{-1 / 2}(\Omega)$. This fact implies immediately the convergence of any Galerkin approximation scheme for (1.1). Such Galerkin schemes with various spline spaces have been studied theoretically as well as practically by several authors (see $[25,15,6,7,16])$.

In contrast to Galerkin methods, convergence proofs for collocation methods for higher-dimensional integral equations are rare. With the exception of second-kind Fredholm integral equations, only a few special classes of integral equations have been analyzed successfully: bisingular integral equations in [10], and higher-dimensional singular convolution operators of order zero in [21] (see also $[11,17])$. In $[17,2,1]$ one finds overviews of the history of collocation methods for partial differential equations and pseudodifferential equations.

Collocation methods for boundary integral equations for two-dimensional boundary value problems, i.e., for integral equations on open or closed curves in $\mathbb{R}^{2}$, have been studied extensively by many authors with many different methods (see [27, 17] for recent summaries).

Prössdorf and Rathsfeld in [18] use the observation that the collocation matrices are related to finite sections of a fixed infinite Toeplitz matrix. Then the symbolic calculus for Toeplitz matrices, based on Fourier series, can be applied. For the case of strongly singular integral operators (translation-invariant pseudodifferential operators of order zero) on cubes in $\mathbb{R}^{m}, m \geq 2$, a related method was used in [21] to prove stability and convergence of piecewise bilinear collocation in the $L^{2}$ norm. In the present paper, we use this idea as a basis for the analysis of the collocation method for the operator $V$ in (1.1), which is a pseudodifferential operator of order -1 . We expect that our approach will work for more general strongly elliptic pseudodifferential equations.

The infinite Toeplitz matrix which we obtain here for the collocation scheme (1.3) is positive definite and symmetric (see Corollary 3.5), and the finite collocation matrices inherit this property. This fact can also be understood by relating the nodal collocation method with piecewise bilinear splines to the Galerkin method with piecewise constant trial and test functions. The matrix elements for both methods are actually the same (see Remark 5.1). Thus, our method is also related to the method of Arnold and Wendland [2], which gives convergence proofs for nodal collocation with odd-order splines for strongly elliptic pseudodifferential equations on smooth curves by reducing the collocation scheme to an equivalent Galerkin scheme.

After proving, in $\S \S 2-4$, the stability and convergence of the collocation method (1.3), we mention in $\S 5$ some generalizations of our scheme, which can easily be treated with the same ideas. In the same way one also proves stability and convergence for the corresponding one-dimensional problem, namely the nodal collocation with piecewise linear trial functions on a uniform grid for the firstkind integral equation on an interval defined by the single-layer potential for the two-dimensional Laplacian (screen problem in $\mathbb{R}^{2}$ ). Generalizations to higher dimensions are also possible.

\section{BASIC NOTATION}

In the previous section, we introduced the domain $\Omega$ and the grid $\square^{N}$. We need the following additional notation for $N \in \mathbb{N}$, 


$$
\omega^{N}:=\left\{k \in \mathbb{Z}^{2} \mid x_{k}^{N} \in \bar{\Omega}\right\}=\left\{k \in \mathbb{Z}^{2}|| k_{j} \mid \leq N, j=1,2\right\} .
$$

A nodal basis for the space $S^{1}\left(\square^{N}\right)$ of piecewise bilinear splines is defined as follows: Let $\phi^{0}(t):=\max \{0,1-|t|\}$ for $t \in \mathbb{R}$, and define for $x \in \mathbb{R}^{2}$

$$
\begin{aligned}
\varphi_{0}^{1}(x) & :=\phi^{0}\left(x_{1}\right) \cdot \phi^{0}\left(x_{2}\right), \\
\varphi_{k}^{N}(x) & :=\varphi_{0}^{1}\left(N\left(x-x_{k}^{N}\right)\right)=\varphi_{0}^{1}(N x-k) .
\end{aligned}
$$

Then $S^{1}\left(\square^{N}\right)=\operatorname{span}\left\{\varphi_{k}^{N} \mid k \in \mathbb{Z}^{2}\right\}$. We need the following $(2 N+1)^{2}$-dimensional subspace of $S^{1}\left(\square^{N}\right)$,

$$
S^{1}\left(\square^{N} \cap \bar{\Omega}\right):=\operatorname{span}\left\{\varphi_{k}^{N} \mid k \in \omega^{N}\right\} .
$$

For any continuous function $f$ on $\mathbb{R}^{2}$, we define the restriction to $\square^{N}$ by

$$
r^{N} f:=\left(f\left(x_{k}^{N}\right)\right)_{k \in \mathbb{Z}^{2}} .
$$

For any sequence $\left(f_{k}\right)_{k \in \mathbb{Z}^{2}}$ we define the piecewise bilinear interpolant by

$$
i^{N}\left(f_{k}\right):=\sum_{k \in \mathbb{Z}^{2}} f_{k} \varphi_{k}^{N}
$$

The system of equations corresponding to the collocation equations (1.3) can be written as

$$
r^{N} V i^{N}\left(u_{k}\right)=r^{N} f \text { on } \omega^{N},
$$

where $\left(u_{k}\right)_{k \in \omega^{N}}$ are the nodal values of $u^{N} \in S^{1}\left(\square^{N} \cap \bar{\Omega}\right)$,

$$
u^{N}=\sum_{k \in \omega^{N}} u_{k} \varphi_{k}^{N}
$$

The matrix elements of the system (2.7) are given by

$$
\left(\left(V \varphi_{k}^{N}\right)\left(x_{k^{\prime}}^{N}\right)\right)_{k, k^{\prime} \in \omega^{N} .}
$$

From the translation invariance of the operator $V(1.1)$ and the definition (2.3) of the basis functions $\varphi_{k}^{N}$ we see that this is a Toeplitz matrix.

Remark 2.1. The collocation points in (2.7) include the nodes on the boundary of $\bar{\Omega}$. The definition of the trial function $u^{N}$ uses these nodes, too. Therefore, the support of $u^{N}$ is not contained in $\Omega$ but in the slightly larger set

$$
\left[-1-\frac{1}{N}, 1+\frac{1}{N}\right] \times\left[-1-\frac{1}{N}, 1+\frac{1}{N}\right] .
$$

By $H^{s}\left(\mathbb{R}^{2}\right) \quad(s \in \mathbb{R})$ we denote the usual Sobolev spaces [13]. For a bounded domain $\mathscr{O}$,

$$
p_{\mathscr{O}}:\left.u \mapsto u\right|_{\mathscr{O}}
$$

is the operator of restriction to $\mathscr{O}$. Then

$$
H^{s}(\mathscr{O}):=\left\{\left.u\right|_{\mathscr{O}} \mid u \in H^{s}\left(\mathbb{R}^{2}\right)\right\}=p_{\mathscr{Q}} H^{s}\left(\mathbb{R}^{2}\right)
$$

and

$$
\widetilde{H}^{s}(\mathscr{O}):=\left\{u \in H^{s}\left(\mathbb{R}^{2}\right) \mid \operatorname{supp} u \subset \overline{\mathscr{O}}\right\} .
$$


Then, with respect to the natural extension of the $L^{2}$ duality,

$$
(f, g):=\int_{\mathbb{R}^{2}} f(x) \overline{g(x)} d x
$$

$\tilde{H}^{s}(\mathscr{O})$ is the dual space of $H^{-s}(\mathscr{O})$.

The basic mapping properties of the operator $V$ in the energy spaces are summarized in the following lemma which is well known (see, e.g., [14, 25]).

Lemma 2.2. (i) The operator $p_{\mathscr{O}} V: \widetilde{H}^{-1 / 2}(\mathscr{O}) \rightarrow H^{1 / 2}(\mathscr{O})$ is an isomorphism.

(ii) The sesquilinear form $(V u, w)$ defines an equivalent inner product on $\widetilde{H}^{-1 / 2}(\mathscr{O})$ : There are constants $c_{1}, c_{2}>0$ such that

$$
c_{1}\|u\|_{\widetilde{H}^{-1 / 2}(\mathscr{O})}^{2} \leq(V u, u) \leq c_{2}\|u\|_{\widetilde{H}^{-1 / 2}(\mathscr{O})}^{2}
$$

for all $u \in \widetilde{H}^{-1 / 2}(\mathscr{O})$. The constants $c_{1}, c_{2}$ depend only on the size of the domain $\mathcal{O}$.

(iii) Let $W:=-\Delta V$ be the operator of the normal derivative of the doublelayer potential. Then the sesquilinear form $(W u, w)$ defines an equivalent inner product on $\widetilde{H}^{1 / 2}(\mathscr{O})$ : There are constants $c_{1}, c_{2}>0$ such that

$$
c_{1}\|u\|_{\widetilde{H}^{1 / 2}(\mathscr{O})}^{2} \leq(W u, u) \leq c_{2}\|u\|_{\widetilde{H}^{1 / 2}(\mathscr{O})}^{2} .
$$

\section{StABility OF THE COLLOCATION METHOD}

Let

$$
\Pi^{N}:=i^{N} r^{N}
$$

be the interpolation projector onto the spline space $S^{1}\left(\square^{N}\right)$. We can write the collocation equations (1.3) in the form

$$
p_{\Omega} \Pi^{N} V u^{N}=p_{\Omega} \Pi^{N} f .
$$

Our aim in this section is the proof of the stability and uniform boundedness of the sequence $\left(p_{\Omega} \Pi^{N} V\right)_{N \in \mathbb{N}}$. Note that the projection $\Pi^{N}$ is not bounded on $H^{1 / 2}$, so that even the uniform boundedness is a nontrivial result.

In the following we will denote by $C$ generic constants independent of $N$.

Theorem 3.1 (Stability). There is a constant $\gamma>0$ such that for all $N \in \mathbb{N}$ and all $w \in S^{1}\left(\square^{N} \cap \bar{\Omega}\right)$ there holds

$$
\left\|p_{\Omega} \Pi^{N} V w\right\|_{H^{1 / 2}(\Omega)} \geq \gamma\|w\|_{H^{-1 / 2}\left(\mathbb{R}^{2}\right)} .
$$

Theorem 3.2 (Uniform boundedness). There is a constant $C \in \mathbb{R}$ such that for all $N \in \mathbb{N}$ and all $w \in S^{1}\left(\square^{N} \cap \bar{\Omega}\right)$ there holds

$$
\left\|p_{\Omega} \Pi^{N} V w\right\|_{H^{1 / 2}(\Omega)} \leq C\|w\|_{H^{-1 / 2}\left(\mathbb{R}^{2}\right)} .
$$

For the proof of these theorems we need some additional tools. First we need the well-known approximation property and inverse inequality for our spline space (see $[4,3,22])$. 
Theorem 3.3. Let $\mathscr{O} \subset \mathbb{R}^{2}$ be a square. (We need the two cases $\mathscr{O}=\Omega$ and $\mathscr{O}=2 \Omega$.)

(i) For any $s \in\left(1, \frac{3}{2}\right)$ there is a constant $C$ such that

$$
\left\|p_{\mathscr{O}} \Pi^{N} v-v\right\|_{H^{1 / 2}(\mathscr{O})} \leq C N^{1 / 2-s}\|v\|_{H^{s}(\mathcal{O})}
$$

for all $v \in H^{s}(\mathscr{O})$, and

$$
\left\|\Pi^{N} v-v\right\|_{H^{1 / 2\left(\mathbb{R}^{2}\right)}} \leq C N^{1 / 2-s}\|v\|_{\widetilde{H}^{s}(\mathscr{O})}
$$

for all $v \in \widetilde{H}^{s}(\mathscr{O})$.

(ii) For any $t \leq s<\frac{3}{2}$ there is a constant $C$ such that

$$
\left\|p_{\mathscr{O}} v\right\|_{H^{s}(\mathscr{O})} \leq C N^{s-t}\left\|p_{\mathscr{O}} v\right\|_{H^{\prime}(\mathscr{O})}
$$

for all $v \in S^{1}\left(\square^{N}\right)$, and

$$
\|v\|_{H^{s}\left(\mathbb{R}^{2}\right)} \leq C N^{s-t}\|v\|_{H^{t}\left(\mathbb{R}^{2}\right)}
$$

for all $v \in S^{1}\left(\square^{N} \cap \overline{\mathscr{O}}\right)$.

Next we introduce discrete Sobolev spaces. Our spaces are related to those studied by Frank [9] and Stephan [24] (see also [20, 19]).

We define a norm in the space of sequences by

$$
\left|\left(u_{k}\right)_{k \in \mathbb{Z}^{2}}\right|_{s, N}:=\left\|i^{N}\left(u_{k}\right)\right\|_{H^{s}\left(\mathbb{R}^{2}\right)} .
$$

The discrete Sobolev space is

$$
h_{N}^{s}\left(\mathbb{Z}^{2}\right):=\left\{\left.\left(u_{k}\right)_{k \in \mathbb{Z}^{2}}||\left(u_{k}\right)\right|_{s, N}<\infty\right\} .
$$

On the grid $\omega^{N}$ we define the finite-dimensional subspace

$$
\tilde{h}_{N}^{s}\left(\omega^{N}\right):=\left\{\left(u_{k}\right)_{k \in \mathbb{Z}^{2}} \in h_{N}^{s}\left(\mathbb{Z}^{2}\right) \mid u_{k}=0 \text { for } k \notin \omega^{N}\right\} .
$$

This is $\mathbb{C}^{M}, M=(2 N+1)^{2}$, with norm $|\cdot|_{s, N}$.

It is easy to see that for $s=0$ we have the norm equivalence

$$
\left|\left(u_{k}\right)\right|_{0, N} \approx N^{-1}\left(\sum_{k \in \mathbb{Z}^{2}}\left|u_{k}\right|^{2}\right)^{1 / 2} .
$$

By “ $\approx$ " we denote the equivalence of norms with constants independent of $N$. Taking (3.12) into account, we define the scalar products in $l^{2}$,

$$
\left(\left(u_{k}\right),\left(v_{k}\right)\right)_{N}:=N^{-2} \sum_{k \in \mathbb{Z}^{2}} u_{k} \overline{v_{k}} .
$$

For Fourier series on the square $Q:=(-\pi, \pi) \times(-\pi, \pi)$ we use the following notation:

$$
\tilde{u}(\xi):=\sum_{k \in \mathbb{Z}^{2}} e^{-i k \cdot \xi} u_{k}=: F\left(u_{k}\right)(\xi)
$$

so we have

$$
u_{k}=(2 \pi)^{-2} \int_{Q} \tilde{u}(\xi) e^{i k \cdot \xi} d \xi
$$


The integral operator $V$ defines two discrete sesquilinear forms:

$$
\left(i^{N}\left(u_{k}\right), V i^{N}\left(v_{k}\right)\right)=\sum_{k, k^{\prime}} u_{k}\left(\varphi_{k}^{N}, V \varphi_{k^{\prime}}^{N}\right) \overline{v_{k^{\prime}}}
$$

and

$$
\left(\left(u_{k}\right), r^{N} V i^{N}\left(v_{k}\right)\right)_{N}=N^{-2} \sum_{k, k^{\prime}} u_{k}\left(V \varphi_{k^{\prime}}^{N}\right)\left(x_{k}^{N}\right) \overline{v_{k^{\prime}}} .
$$

The form (3.16) defines a matrix which corresponds to a Galerkin method for the equation $V u=f$. The form (3.17) corresponds to our collocation method (1.3). More precisely, the collocation scheme (1.3) can be considered as a finite section method for the infinite Toeplitz matrix defined by (3.17). The diagonalization of the infinite Toeplitz matrices via Fourier series leads to the following representations of the bilinear forms (3.16), (3.17).

Lemma 3.4. Let $\tilde{u}=F\left(u_{k}\right)$ and $\tilde{v}=F\left(v_{k}\right)$ with $\left(u_{k}\right),\left(v_{k}\right) \in l^{1}\left(\mathbb{Z}^{2}\right)$. Then

(i) $\left(i^{N}\left(u_{k}\right), V i^{N}\left(v_{k}\right)\right)=N^{-3} \int_{Q} \lambda^{G}(\xi) \tilde{u}(\xi) \overline{\tilde{v}(\xi)} d \xi$;

(ii) $\left(\left(u_{k}\right), r^{N} V i^{N}\left(v_{k}\right)\right)_{N}=N^{-3} \int_{Q} \lambda^{C}(\xi) \tilde{u}(\xi) \overline{\tilde{v}(\xi)} d \xi$.

With the operator $W$ defined in Lemma 2.2(iii), we have

(iii) $\left(i^{N}\left(u_{k}\right), W i^{N}\left(v_{k}\right)\right)=N^{-1} \int_{Q} \lambda^{W, G}(\xi) \tilde{u}(\xi) \overline{\tilde{v}(\xi)} d \xi$.

Here the functions $\lambda^{G}, \lambda^{C}$, and $\lambda^{W, G}$ are defined by

$$
\begin{aligned}
\lambda^{G}(\xi) & =2^{7} \cdot(2 \pi)^{-2} \sum_{r \in \mathbb{Z}^{2}}|\xi+2 \pi r|^{-1} \prod_{j=1}^{2} \frac{\sin ^{4}\left(\xi_{j} / 2\right)}{\left(\xi_{j}+2 \pi r_{j}\right)^{4}}, \\
\lambda^{C}(\xi) & =8 \cdot(2 \pi)^{-2} \sum_{r \in \mathbb{Z}^{2}}|\xi+2 \pi r|^{-1} \prod_{j=1}^{2} \frac{\sin ^{2}\left(\xi_{j} / 2\right)}{\left(\xi_{j}+2 \pi r_{j}\right)^{2}}, \\
\lambda^{W, G}(\xi) & =2^{7} \cdot(2 \pi)^{-2} \sum_{r \in \mathbb{Z}^{2}}|\xi+2 \pi r| \prod_{j=1}^{2} \frac{\sin ^{4}\left(\xi_{j} / 2\right)}{\left(\xi_{j}+2 \pi r_{j}\right)^{4}} .
\end{aligned}
$$

Proof. For functions $u \in C_{0}^{\infty}\left(\mathbb{R}^{2}\right)$ we define the Fourier transform by

$$
\hat{u}(\xi):=\int_{\mathbb{R}^{2}} e^{-i \xi \cdot x} u(x) d x .
$$

It is well known (see [8]) that the operators $V$ and $W$ have the Fourier representation

$$
\begin{aligned}
& V u(x)=\frac{1}{2}(2 \pi)^{-2} \int_{\mathbb{R}^{2}} e^{i \xi \cdot x}|\xi|^{-1} \hat{u}(\xi) d \xi, \\
& W u(x)=\frac{1}{2}(2 \pi)^{-2} \int_{\mathbb{R}^{2}} e^{i \xi \cdot x}|\xi| \hat{u}(\xi) d \xi .
\end{aligned}
$$

From the definition (2.3) we obtain

$$
\widehat{\varphi_{k}^{N}}(\xi)=N^{-2} e^{-i \xi \cdot k / N} \widehat{\varphi_{0}^{1}}\left(\frac{\xi}{N}\right) .
$$

Parseval's formula yields

$$
\begin{aligned}
& \left(\varphi_{k}^{N}, V \varphi_{k^{\prime}}^{N}\right)=\frac{1}{2}(2 \pi)^{-2} \int_{\mathbb{R}^{2}}|\xi|^{-1} \widehat{\varphi_{k}^{N}}(\xi) \widehat{\widehat{\varphi_{k^{\prime}}^{N}}(\xi)} d \xi \\
& =N^{-3} \int_{Q} e^{i\left(k^{\prime}-k\right) \cdot \xi} \lambda^{G}(\xi) d \xi,
\end{aligned}
$$


with

$$
\lambda^{G}(\xi)=\left.\frac{1}{2}(2 \pi)^{-2} \sum_{r \in \mathbb{Z}^{2}}|\xi+2 \pi r|^{-1} \widehat{\mid \varphi_{0}^{1}}(\xi+2 \pi r)\right|^{2} .
$$

From (2.2) we compute the Fourier transform

$$
\widehat{\varphi_{0}^{1}}(\xi)=16 \prod_{j=1}^{2} \frac{\sin ^{2}\left(\xi_{j} / 2\right)}{\xi_{j}^{2}}
$$

Inserting this in (3.24), we find (3.18a), and from (3.16) we obtain

$$
\begin{aligned}
\left(i^{N}\left(u_{k}\right), V i^{N}\left(v_{k}\right)\right) & =N^{-3} \sum_{k, k^{\prime} \in \mathbb{Z}^{2}} u_{k} \overline{v_{k^{\prime}}} \int_{Q} e^{i\left(k^{\prime}-k\right) \cdot \xi} \lambda^{G}(\xi) d \xi \\
& =N^{-3} \int_{Q} \tilde{u}(\xi) \overline{\tilde{v}(\xi)} \lambda^{G}(\xi) d \xi
\end{aligned}
$$

This proves (i). For a more detailed computation, see [21]. For the bilinear form (3.17), we compute with (3.20) the matrix element

$$
\begin{aligned}
V \varphi_{k^{\prime}}^{N}\left(x_{k}^{N}\right) & =\frac{1}{2}(2 \pi)^{-2} \int_{\mathbb{R}^{2}} e^{i \xi \cdot x_{k}^{N}}|\xi|^{-1} \widehat{\varphi_{k^{\prime}}^{N}}(\xi) d \xi \\
& =N^{-1} \int_{Q} e^{i\left(k-k^{\prime}\right) \cdot \xi} \lambda^{C}(-\xi) d \xi,
\end{aligned}
$$

with $\lambda^{C}$ defined by $(3.18 \mathrm{~b})$. This proves (ii).

For (iii), we observe that according to (3.21), we have to replace the multiplier $|\xi|^{-1}$ in $(3.23)$ by $|\xi|$.

From the definitions $(3.18 \mathrm{a}, \mathrm{b}, \mathrm{c})$ we see immediately that the functions $\lambda^{G}, \lambda^{C}$, and $\lambda^{W, G}$ are positive $C^{\infty}$ functions on $\bar{Q} \backslash\{0\}$, and that $\lambda^{G}$ and $\lambda^{C}$ behave like $\mathscr{O}\left(|\xi|^{-1}\right)$ at $\xi=0$, whereas $\lambda^{W, G}(\xi)=\mathscr{O}(|\xi|)$ near $\xi=0$. Therefore, $\lambda^{G}, \lambda^{C}$, and $\left(\lambda^{W, G}\right)^{-1}$ define equivalent weights on $Q$, i.e., there exist constants $c_{1}, c_{2}, c_{3}>0$ such that

$$
c_{1} \lambda^{G}(\xi) \leq c_{2} \lambda^{C}(\xi) \leq\left(\lambda^{W, G}(\xi)\right)^{-1} \leq c_{3} \lambda^{G}(\xi)
$$

for all $\xi \in \bar{Q} \backslash\{0\}$.

Corollary 3.5. For any $N \in \mathbb{N}$, the collocation matrix in (1.3) is positive definite and symmetric. The collocation equations are always uniquely solvable.

Corollary 3.6. (i) Both quadratic forms (3.16) and (3.17) define norms on $\tilde{h}_{N}^{-1 / 2}\left(\omega^{N}\right)$ which are equivalent to the $\left.\right|_{-1 / 2, N}$ norm (3.9) with constants not depending on $N$.

(ii) Let $\mathscr{O} \subset \mathbb{R}^{2}$ be a bounded domain. For sequences $\left(v_{k}\right)$ with supp $i^{N}\left(v_{k}\right) \subset$ - we have the norm equivalence

$$
\left|\left(v_{k}\right)\right|_{1 / 2, N}^{2} \approx N^{-1} \int_{Q}|\tilde{v}(\xi)|^{2}\left(\lambda^{G}(\xi)\right)^{-1} d \xi
$$

Proof. The equivalence of the norms corresponding to the sesquilinear forms (3.16) and (3.17) follows directly from Lemma 3.4(i) and (ii) and (3.27). The 
equivalence with the $|\cdot|_{-1 / 2, N}$ norm follows from Lemma 2.2(ii). The equivalence (3.28) follows from Lemma 2.2(iii) together with (3.27) and Lemma 3.4(iii).

Proof of Theorem 3.1. Let $\widehat{\Omega}=2 \Omega=(-2,2) \times(-2,2)$. We need an extension operator $L$ from $H^{s}(\Omega)$ to $\widetilde{H}^{s}(\widehat{\Omega})$. It is well known [23] that such an operator exists as a continuous linear operator

$$
L: H^{s}(\Omega) \rightarrow \widetilde{H}^{s}(\widehat{\Omega}) \text { for all } s \geq 0 .
$$

We can assume that $L$ is given in such a way that $\operatorname{supp} \Pi^{N} L v \subset \widehat{\Omega}$ for all $N$ and all $v \in H^{s}(\Omega)$. We show first that there is a constant $C$ independent of $N$ such that

$$
\left\|\Pi^{N} L p_{\Omega} u\right\|_{\widetilde{H}^{1 / 2}(\widehat{\Omega})} \leq C\left\|p_{\Omega} u\right\|_{H^{1 / 2}(\Omega)}
$$

holds for all $u \in S^{1}\left(\square^{N}\right)$. Indeed, we have

$$
\left\|\Pi^{N} L p_{\Omega} u\right\|_{\widetilde{H}^{1 / 2}(\widehat{\Omega})} \leq\left\|L p_{\Omega} u\right\|_{\widetilde{H}^{1 / 2}(\widehat{\Omega})}+\left\|\left(I-\Pi^{N}\right) L p_{\Omega} u\right\|_{\widetilde{H}^{1 / 2}(\widehat{\Omega})} .
$$

The first term on the right-hand side in (3.31) is bounded by $C\left\|p_{\Omega} u\right\|_{H^{1 / 2}(\Omega)}$ according to (3.29). For the second term we use the approximation property (3.6) and the inverse inequality (3.7) and obtain for $s \in\left(1, \frac{3}{2}\right)$

$$
\begin{aligned}
\left\|\left(I-\Pi^{N}\right) L p_{\Omega} u\right\|_{\widetilde{H}^{1 / 2}(\widehat{\Omega})} & \leq C N^{1 / 2-s}\left\|L p_{\Omega} u\right\|_{\widetilde{H}^{s}(\widehat{\Omega})} \\
& \leq C N^{1 / 2-s}\left\|p_{\Omega} u\right\|_{H^{s}(\Omega)} \leq C\left\|p_{\Omega} u\right\|_{H^{1 / 2}(\Omega)} .
\end{aligned}
$$

Next we show that there is a constant independent of $N$ such that

$$
\left|\left(\left(u_{k}\right),\left(v_{k}\right)\right)_{N}\right| \leq C\left|\left(u_{k}\right)\right|_{-1 / 2, N} \cdot\left|\left(v_{k}\right)\right|_{1 / 2, N}
$$

for all sequences $\left(u_{k}\right),\left(v_{k}\right)$ with $\operatorname{supp} i^{N}\left(u_{k}\right) \subset \widehat{\Omega}$, supp $i^{N}\left(v_{k}\right) \subset \widehat{\Omega}$. By Parseval's formula, we have

$$
\begin{aligned}
\left|\left(\left(u_{k}\right),\left(v_{k}\right)\right)_{N}\right|= & N^{-2}(2 \pi)^{-2}\left|\int_{Q} \tilde{u}(\xi) \overline{\tilde{v}(\xi)} d \xi\right| \\
\leq & \left(N^{-3} \int_{Q}|\tilde{u}(\xi)|^{2} \lambda^{G}(\xi) d \xi\right)^{1 / 2} \\
& \cdot\left(N^{-1} \int_{Q}|\tilde{v}(\xi)|^{2}\left(\lambda^{G}(\xi)\right)^{-1} d \xi\right)^{1 / 2} .
\end{aligned}
$$

The first factor on the right-hand side is bounded by $C\left|\left(u_{k}\right)\right|_{-1 / 2, N}$ according to Corollary 3.6(i), and the second factor is bounded by $C\left|\left(v_{k}\right)\right|_{1 / 2, N}$ according to (3.28). Thus (3.32) is shown.

Combining the estimates (3.30), (3.32), and Corollary 3.6(i), we obtain for $w=i^{N}\left(w_{k}\right) \in S^{1}\left(\square^{N} \cap \bar{\Omega}\right)$

$$
\begin{aligned}
& \left|\left(w_{k}\right)\right|_{-1 / 2, N} \cdot\left\|p_{\Omega} \Pi^{N} V w\right\|_{H^{1 / 2}(\Omega)} \geq C\left\|\Pi^{N} L p_{\Omega} \Pi^{N} V w\right\|_{\widetilde{H}^{1 / 2}(\widehat{\Omega})} \cdot\left|\left(w_{k}\right)\right|_{-1 / 2, N} \\
& \quad=C\left|r^{N} L p_{\Omega} \Pi^{N} V w\right|_{1 / 2, N} \cdot\left|\left(w_{k}\right)\right|_{-1 / 2, N} \geq C\left|\left(\left(w_{k}\right), r^{N} L p_{\Omega} \Pi^{N} V w\right)_{N}\right| \\
& \quad=C\left|\left(\left(w_{k}\right), r^{N} V i^{N}\left(w_{k}\right)\right)_{N}\right| \geq C\left|\left(w_{k}\right)\right|_{-1 / 2, N}^{2} . \quad \square
\end{aligned}
$$

Proof of Theorem 3.2. This follows by the same arguments as (3.30) above. 


\section{Convergence}

In this section we prove the following convergence theorem.

Theorem 4.1. Let $f \in H^{1+\varepsilon}(\Omega)$ with some $\varepsilon>0$ and $u \in \widetilde{H}^{-1 / 2}(\Omega)$ be the solution of the integral equation (1.1). Then for any $N \in \mathbb{N}$, the collocation equations (1.3) have a unique solution $u^{N} \in S^{1}\left(\square^{N} \cap \bar{\Omega}\right)$. For any $\eta>0$ there is a constant $C \geq 0$ independent of $N$ such that

$$
\left\|u-u^{N}\right\|_{H^{-1 / 2}\left(\mathbb{R}^{2}\right)} \leq C N^{-1 / 2+\eta}\|f\|_{H^{1+\varepsilon}(\Omega)} .
$$

Proof. Let $P^{N}$ be the $L^{2}$ orthogonal projector onto $S^{1}\left(\square^{N} \cap \bar{\Omega}\right)$. It is well known that $P^{N}$ is uniformly bounded in the $H^{s}\left(\mathbb{R}^{2}\right)$ norms for $|s|<\frac{3}{2}$ and that there holds the approximation property

$$
\left\|v-P^{N} v\right\|_{H^{-1 / 2\left(\mathbb{R}^{2}\right)}} \leq C N^{-1 / 2+\eta}\|v\|_{H^{-\eta}\left(\mathbb{R}^{2}\right)}
$$

for all $v \in \widetilde{H}^{-\eta}(\Omega)$. Then we have

$$
\left\|u-u^{N}\right\|_{H^{-1 / 2}\left(\mathbb{R}^{2}\right)} \leq\left\|u-P^{N} u\right\|_{H^{-1 / 2}\left(\mathbb{R}^{2}\right)}+\left\|P^{N} u-u^{N}\right\|_{H^{-1 / 2}\left(\mathbb{R}^{2}\right)} .
$$

We know that $u \in H^{-\eta}(\Omega)$ holds for any $\eta>0$ (see [5]). Therefore, (4.1) gives for the first term on the right-hand side of (4.2) the estimate

$$
\left\|u-P^{N} u\right\|_{H^{-1 / 2}\left(\mathbb{R}^{2}\right)} \leq C N^{-1 / 2+\eta}\|u\|_{H^{-\eta}(\Omega)} \leq C N^{-1 / 2+\eta}\|f\|_{H^{1-\eta}(\Omega)} .
$$

For the second term on the right-hand side of (4.2) we estimate further, using the stability estimate (3.3) and Lemma 3.3:

$$
\begin{aligned}
\left\|P^{N} u-u^{N}\right\|_{H^{-1 / 2}\left(\mathbb{R}^{2}\right)} & \leq C\left\|p_{\Omega} \Pi^{N} V\left(P^{N} u-u^{N}\right)\right\|_{H^{1 / 2}(\Omega)} \\
& =C\left\|p_{\Omega} \Pi^{N} V P^{N} u-p_{\Omega} \Pi^{N} f\right\|_{H^{1 / 2}(\Omega)} \\
& \leq C\left\|p_{\Omega} \Pi^{N} V P^{N} u-p_{\Omega} f\right\|_{H^{1 / 2}(\Omega)}+\left\|p_{\Omega}\left(f-\Pi^{N} f\right)\right\|_{H^{1 / 2}(\Omega)} .
\end{aligned}
$$

The last term can be estimated with (3.5),

$$
\left\|p_{\Omega}\left(f-\Pi^{N} f\right)\right\|_{H^{1 / 2}(\Omega)} \leq C N^{1 / 2-(1+\varepsilon)}\|f\|_{H^{1+\varepsilon}(\Omega)} .
$$

Finally, we have

$$
\begin{aligned}
\left\|p_{\Omega} \Pi^{N} V P^{N} u-p_{\Omega} f\right\|_{H^{1 / 2}(\Omega)} \\
\quad \leq\left\|p_{\Omega} \Pi^{N} V P^{N} u-p_{\Omega} V P^{N} u\right\|_{H^{1 / 2}(\Omega)}+\left\|p_{\Omega}\left(V P^{N} u-V u\right)\right\|_{H^{1 / 2}(\Omega)} \\
\quad \leq C\left(N^{-1 / 2-\varepsilon}\left\|V P^{N} u\right\|_{H^{1+\varepsilon}(\Omega)}+\left\|P^{N} u-u\right\|_{H^{-1 / 2}\left(\mathbb{R}^{2}\right)}\right) \\
\quad \leq C\left(N^{-1 / 2-\varepsilon}\left\|P^{N} u\right\|_{H^{\varepsilon}\left(\mathbb{R}^{2}\right)}+N^{-1 / 2-\varepsilon}\|f\|_{H^{1+\varepsilon}(\Omega)}\right) \\
\quad \leq C\left(N^{-1 / 2+\eta}\left\|P^{N} u\right\|_{H^{-\eta}\left(\mathbb{R}^{2}\right)}+N^{-1 / 2-\varepsilon}\|f\|_{H^{1+\varepsilon}(\Omega)}\right) \\
\quad \leq C\left(N^{-1 / 2+\eta}\|u\|_{H^{-\eta}\left(\mathbb{R}^{2}\right)}+N^{-1 / 2-\varepsilon}\|f\|_{H^{1+\varepsilon}(\Omega)}\right) \\
\quad \leq C N^{-1 / 2+\eta}\|f\|_{H^{1+\varepsilon}(\Omega)} .
\end{aligned}
$$

\section{Generalization}

In this final section we want to mention briefly some generalizations of the collocation method (1.3) for which our present approach is also applicable.

The first generalization concerns the domain $\Omega$. Instead of the square, we can consider any polygonal domain which is a union of a finite number of cells 
of some rectangular grid $\Delta_{h_{1}, h_{2}}:=\left\{\left(k_{1} h_{1}, k_{2} h_{2}\right) \mid k \in \mathbb{Z}^{2}\right\}$. The grid $\square^{N}$ has to be replaced by $\frac{1}{N} \Delta_{h_{1}, h_{2}}$. It is easy to see that the results of $\S \S 3$ and 4 remain valid for this case.

The second generalization concerns the splines which are used as trial functions. We can prove the stability and convergence results of the previous sections for splines which are tensor products of univariate splines of odd order. Since the statements of Theorems 3.1, 3.2, and 4.1 remain the same in this case, we only mention here the change in the essential part of the proof of Theorem 3.1, namely statement (ii) of Lemma 3.4. If we use tensor products of $B$-splines of degrees $d_{1}$ and $d_{2}$ as a basis, then the symbol $\lambda^{C}(\xi)$ of the collocation matrix is changed to

$$
\lambda^{C}(\xi)=(2 \pi)^{-2} \cdot 2^{d_{1}+d_{2}+1} \sum_{r \in \mathbb{Z}^{2}}|\xi+2 \pi r|^{-1} \prod_{j=1}^{2}\left(\frac{\sin \left(\xi_{j} / 2\right)}{\xi_{j}+2 \pi r_{j}}\right)^{d_{j}+1} .
$$

For the interpolation projection $\Pi^{N}$ in Theorems 3.1 and 3.2, we use piecewise bilinear functions also in this more general case. It is clear that the new function $\lambda^{C}$ in (5.1) is a weight function on $\bar{Q}$ which is equivalent to the functions defined in (3.18a) and (3.18b).

Remark 5.1. If we compute the symbol $\lambda^{G}$ of the Galerkin matrix with tensor product splines, we obtain

$$
\lambda^{G}(\xi)=(2 \pi)^{-2} \cdot 2^{2\left(d_{1}+d_{2}\right)+3} \sum_{r \in \mathbb{Z}^{2}}|\xi+2 \pi r|^{-1} \prod_{j=1}^{2}\left(\frac{\sin \left(\xi_{j} / 2\right)}{\xi_{j}+2 \pi r_{j}}\right)^{2 d_{j}+2} .
$$

Comparing this formula for $d_{1}=d_{2}=0$ with $(3.18 \mathrm{~b})$, we see that the matrix elements for the piecewise bilinear collocation method are the same as those for the piecewise constant Galerkin method.

The last generalization which we want to mention concerns the integral operator $V$. Instead of $V$ as defined in (1.1), we can use a convolution operator $A$ which has a Fourier representation

$$
\widehat{A u}(\xi)=\sigma_{A}(\xi) \hat{u}(\xi) .
$$

We make the assumption that $A$ is strongly elliptic of order -1 , i.e.,

$$
\sigma_{A}(t \xi)=t^{-1} \sigma_{A}(\xi) \text { for all } \xi \in \mathbb{R}^{2} \backslash\{0\}, t>0,
$$

$|\xi| \cdot \sigma_{A}(\xi)$ is bounded, and

$$
\operatorname{Re} \sigma_{A}(\xi) \geq \gamma>0 \text { for all } \xi \in \mathbb{R}^{2} \text { with }|\xi|=1 .
$$

Then we can prove the stability result of Theorem 3.1 exactly in the same way as for $V$ above. The symbol $\lambda^{C}$ of the collocation matrix is then changed to

$$
\lambda^{A}, C(\xi)=16 \cdot(2 \pi)^{-2} \sum_{r \in \mathbb{Z}^{2}} \sigma_{A}(\xi+2 \pi r) \prod_{j=1}^{2} \frac{\sin ^{2}\left(\xi_{j} / 2\right)}{\left(\xi_{j}+2 \pi r_{j}\right)^{2}} .
$$

From the assumption (5.5) it follows that

$$
\operatorname{Re} \lambda^{A, C}(\xi) \geq 2 \gamma \lambda^{C}(\xi) \text { for all } \xi \in \bar{Q},
$$

which is an essential part of the proof of the stability estimate. 
Finally, we want to mention that the stability result also holds for strongly elliptic systems of convolution operators of order -1 , for instance for the operator defined by the single-layer potential of the equations of linear threedimensional elasticity theory.

\section{BIBLIOGRAPHY}

1. D. N. Arnold and J. Saranen, On the asymptotic convergence of spline collocation methods for partial differential equations, SIAM J. Numer. Anal. 21 (1984), 459-472.

2. D. N. Arnold and W. L. Wendland, On the asymptotic convergence of collocation methods, Math. Comp. 41 (1983), 349-381.

3. J. Aubin, Approximation of elliptic boundary value problems, Wiley-Interscience, New York, 1972.

4. I. Babuška and A. K. Aziz, Survey lectures on the mathematical foundation of the finite element method, The Mathematical Foundation of the Finite Element Method with Applications to Partial Differential Equations (A. K. Aziz, ed.), Academic Press, New York, 1972, pp. 3-359.

5. M. Dauge, Elliptic boundary value problems in corner domains-smoothness and asymptotics of solutions, Lecture Notes in Math., vol. 1341, Springer-Verlag, Berlin, 1988.

6. V. J. Ervin and E. P. Stephan, Experimental convergence of boundary element methods for the capacity of the electrified plate, Boundary Elements IX (C. A. Brebbia, W. L. Wendland, and G. Kuhn, eds.), vol. 1, Springer-Verlag, Berlin, 1987, pp. 167-175.

7. V. J. Ervin, E. P. Stephan, and S. Abou El-Seoud, An improved boundary element method for the charge density of a thin electrified plate in $\mathbb{R}^{3}$, Math. Meth. Appl. Sci. 13 (1990), 291-303.

8. G. I. Ėskin, Boundary value problems for elliptic pseudodifferential equations, Transl. Math. Monographs, vol. 52, Amer. Math. Soc., Providence, RI, 1981.

9. L. S. Frank, Spaces of network functions, Math. USSR-Sb. 15 (1971), 183-226.

10. R. Hagen and B. Silbermann, On finite element collocation for bisingular integral equations, Appl. Anal. 19 (1985), 117-135.

11. G. C. Hsiao and S. Prössdorf, On spline collocation for multidimensional singular integral equations (to appear).

12. M. A. Jaswon and G. T. Symm, Integral equation methods in potential theory and elastostatics, Academic Press, London, 1977.

13. J. L. Lions and E. Magenes, Nonhomogeneous boundary value problems and applications, vol. 1, Springer-Verlag, Berlin, 1972.

14. J.-C. Nédélec, Equations intégrales associées aux problèmes aux limites elliptiques dans des domaines de $\mathbb{R}^{3}$, Analyse Mathématique et Calcul Numérique pour les Sciences et les Techniques (R. Dautray and J.-L. Lions, eds.), Chapters XI-XIII, Masson, Paris, 1988.

15. F. Penzel, Error estimates for a discretized Galerkin method for a boundary integral equation in two dimensions, THD-Preprint 1276, Technische Hochschule Darmstadt, 1990.

16. T. von Petersdorff, Randwertprobleme der Elastizitätstheorie für Polyeder-Singularitäten und Approximation mit Randelementmethoden, Thesis, Technische Hochschule Darmstadt, 1989.

17. S. Prössdorf, Numerische Behandlung singulärer Integralgleichungen, Z. Angew. Math. Mech. 69 (1989), T5-T13.

18. S. Prössdorf and A. Rathsfeld, A spline collocation method for singular integral equations with piecewise continuous coefficients, Integral Equations Operator Theory 7 (1984), 536560.

19. G. Schmidt, Spline collocation for singular integro-differential equations over $(0,1), \mathrm{Nu}-$ mer. Math. 50 (1987), 337-352.

20. G. Schmidt and H. Strese, The convergence of a direct BEM for the plane mixed boundary value problem of the Laplacian, Numer. Math. 54 (1988), 145-165. 
21. R. Schneider, Stability of a collocation method for strongly elliptic multidimensional singular integral equations, Numer. Math. 58 (1991), 855-873.

22. L. L. Schumaker, Spline functions: Basic theory, Wiley, New York, 1981.

23. E. M. Stein, Singular integrals and differentiability properties of functions, Princeton Univ. Press, Princeton, NJ, 1970.

24. E. P. Stephan, Differenzenapproximationen von Pseudo-Differentialoperatoren, Thesis, Technische Hochschule Darmstadt, 1975.

25. __ Boundary integral equations for screen problems in $\mathbb{R}^{3}$, Integral Equations Operator Theory 10 (1987), 236-257.

26. W. L. Wendland, On some mathematical aspects of boundary element methods for elliptic problems, Mathematics of Finite Elements and Applications V (J. Whiteman, ed.), Academic Press, London, 1985, pp. 193-227.

27. _ Strongly elliptic boundary integral equations, The State of the Art in Numerical Analysis (A. Iserles and M. J. D. Powell, eds.), Clarendon Press, Oxford, 1987, pp. 511-562.

(M. Costabel) CeReMaB, Université Bordeaux 1, 351 Cours de la Libération, 33405 Talence Cedex, France

(F. Penzel and R. Schneider) Fachbereich Mathematik, Technische Hochschule DarmStadt, Schlossgartenstr. 7, D-6100 Darmstadt, Germany 